\title{
On the Krull dimension of rings of continuous semialgebraic functions
}

\author{
José F. Fernando and José M. Gamboa
}

\begin{abstract}
Let $R$ be a real closed field, $\mathcal{S}(M)$ the ring of continuous semialgebraic functions on a semialgebraic set $M \subset R^{m}$ and $\mathcal{S}^{*}(M)$ its subring of continuous semialgebraic functions that are bounded with respect to $R$. In this work we introduce semialgebraic pseudo-compactifications of $M$ and the semialgebraic depth of a prime ideal $\mathfrak{p}$ of $\mathcal{S}(M)$ in order to provide an elementary proof of the finiteness of the Krull dimensions of the rings $\mathcal{S}(M)$ and $\mathcal{S}^{*}(M)$ for an arbitrary semialgebraic set $M$. We are inspired by the classical way to compute the dimension of the ring of polynomial functions on a complex algebraic set without involving the sophisticated machinery of real spectra. We show $\operatorname{dim}(\mathcal{S}(M))=\operatorname{dim}\left(\mathcal{S}^{*}(M)\right)=\operatorname{dim}(M)$ and prove that in both cases the height of a maximal ideal corresponding to a point $p \in M$ coincides with the local dimension of $M$ at $p$. In case $\mathfrak{p}$ is a prime $z$-ideal of $\mathcal{S}(M)$, its semialgebraic depth coincides with the transcendence degree of the real closed field $\mathrm{qf}(\mathcal{S}(M) / \mathfrak{p})$ over $R$.
\end{abstract}

\section{Introduction}

Let $R$ be a real closed field. A subset $M \subset R^{m}$ is semialgebraic if it has a description by a finite boolean combination of polynomial equations and inequalities, which we will call a semialgebraic description. A map $f: M \rightarrow N$ is semialgebraic if its graph is a semialgebraic set (in particular $M$ and $N$ are semialgebraic sets). In case $N=R$, we say that $f: M \rightarrow R$ is a semialgebraic function. The sum and product defined pointwise endow the set $\mathcal{S}(M)$ of continuous semialgebraic functions on $M$ with a natural structure of a unital commutative ring. It is obvious that the subset $\mathcal{S}^{*}(M)$ of continuous semialgebraic functions on $M$ that are bounded with respect to $R$ is a real subalgebra of $\mathcal{S}(M)$.

In the following we denote either $\mathcal{S}(M)$ or $\mathcal{S}^{*}(M)$ by $\mathcal{S}^{\diamond}(M)$ if the involved statements or arguments are valid for both rings simultaneously. For instance,

Mathematics Subject Classification (2010): Primary 14P10, 54C30; Secondary 12D15, 13E99.

Keywords: Continuous semialgebraic function, bounded continuous semialgebraic function, $z$-ideal, semialgebraic depth, Krull dimension, local dimension, transcendence degree, real closed field. 
if $p \in M$, we denote the maximal ideal of all functions in $\mathcal{S}^{\diamond}(M)$ vanishing at $p$ with $\mathfrak{m}_{p}^{\diamond}$. For each $f \in \mathcal{S}^{\diamond}(M)$ we denote its zero set with $Z(f)$.

The rings $\mathcal{S}^{\diamond}(M)$ are particular cases of the so-called real closed rings introduced by Schwartz [17] in the ' 80 s of the last century. The theory of real closed rings has been deeply developed until now in a fruitful attempt to establish new foundations for semialgebraic geometry with relevant interconnections with model theory, see the results of Cherlin-Dickmann [6], [7], Schwartz [17], [18], [19], [20], Schwartz with Prestel, Madden and Tressl [16], [22], [23] and Tressl [24], [25], [26]. This theory, which vastly generalizes the classical techniques concerning the semialgebraic spaces of Delfs-Knebusch [10], provides a powerful machinery to approach problems about certain rings of real valued functions and contributes to achieve a better understanding of the algebraic properties of such rings and the topological properties of their spectra.

In this work we provide an elementary geometric proof of the fact that the rings $\mathcal{S}^{\diamond}(M)$ have finite Krull dimension and that it is equal to the dimension of $M$ without involving the sophisticated machinery of real spectra.

Although they are neither Noetherian nor enjoy primary decomposition properties, these rings are closer to polynomial rings than to classical rings of continuous functions. For example, the Lebesgue dimension of $\mathbb{R}$ is 1 (see Problem 16F in [15]) while the Krull dimension of the $\operatorname{ring} \mathcal{C}(\mathbb{R})$ of real valued continuous functions on $\mathbb{R}$ is infinite, see Problem 14I in [15].

In the polynomial context over an algebraically closed field $C$, Hilbert's Nullstellensatz assures that each radical ideal of $C\left[\mathrm{x}_{1}, \ldots, \mathrm{x}_{m}\right]$ is the ideal of all functions vanishing identically on a certain algebraic subset of $C^{m}$. When handling chains of prime ideals in rings of continuous semialgebraic functions, we are nearer to the polynomial case over an algebraically closed field than to the polynomial case having coefficients in a real closed field. This is why we follow similar guidelines to those involved in the proof that the Krull dimension of a ring of polynomial functions on an algebraic set $Z \subset C^{m}$ coincides with the topological dimension of $Z$. The key is the following: if $\mathfrak{P}_{1} \subsetneq \mathfrak{P}_{2}$ are two prime ideals of $C[\mathrm{x}]$, the dimension of the zero set of $\mathfrak{P}_{2}$ is strictly smaller than the one of $\mathfrak{P}_{1}$ (see Lemma 2.2 for the counterpart of this property in our setting). The dimension is the invariant that bounds the number of possible jumps in a chain of prime ideals.

The common zero set $Z(\mathfrak{p})$ of the continuous semialgebraic functions in a prime ideal $\mathfrak{p}$ of $\mathcal{S}(M)$ is either empty or a point, so it makes no sense to work with its dimension. We substitute it by the semialgebraic depth of $\mathfrak{p}[11]$, that we define as

$$
\mathrm{d}_{M}(\mathfrak{p}):=\min \{\operatorname{dim}(Z(f)): f \in \mathfrak{p}\}
$$

Of course, in the real polynomial case the corresponding semialgebraic depth of a prime ideal equals the dimension of the zero set of the ideal.

The main results proved in this article are stated next.

Theorem 1.1 (Dimension). The Krull dimensions of the rings $\mathcal{S}(M)$ and $\mathcal{S}^{*}(M)$ coincide and are both equal to the topological dimension of $M$. 
Carral and Coste proved the equality $\operatorname{dim}(\mathcal{S}(M))=\operatorname{dim}(M)$ for a locally closed semialgebraic set $M$ in [5] (see also [13], [19], and [21]) by proving that the real spectrum of $\mathcal{S}(M)$ is homeomorphic to the constructible subset $\widetilde{M}$ of the real spectrum of the ring of polynomial functions on $R^{m}$ associated to $M$ (see Chapter 7 of [2] for the technicalities concerning the real spectrum). Gamboa-Ruiz extended this equality to an arbitrary semialgebraic set in [14] using strong properties of the real spectrum of excellent rings and some results of the theory of real closed rings (see [19]).

As far as we know, the equality $\operatorname{dim}\left(\mathcal{S}^{*}(M)\right)=\operatorname{dim}(M)$ was unknown. The crucial tool to approach the previous equality and to extend the results from the locally closed case to the general case is the use of semialgebraic pseudo-compactifications of $M$, which are studied in Section 3. One main property is the following: $\mathcal{S}^{*}(M)$ is the direct limit of the rings $\mathcal{S}(X)$, where $X$ runs over the semialgebraic pseudocompactifications of $M$ (see 3.A.4).

Along this work we denote by $\mathrm{ht}(\mathfrak{p})$ the height of a prime ideal $\mathfrak{p}$, and by $\mathrm{qf}(A)$ the quotient field of the domain $A$.

Theorem 1.2 (Local dimension). Let $p \in M$ and $\mathfrak{m}_{p}^{\diamond}$ be the maximal ideal of $\mathcal{S}^{\diamond}(M)$ associated to $p$. Then $\mathrm{ht}\left(\mathfrak{m}_{p}^{\circ}\right)$ equals the local dimension d of $M$ at $p$. Moreover, there exists a chain of prime ideals $\mathfrak{p}_{0} \subsetneq \cdots \subsetneq \mathfrak{p}_{d}:=\mathfrak{m}_{p}^{\diamond}$ such that the transcendence degree of the real closed field $\mathrm{qf}\left(\mathcal{S}^{\diamond}(M) / \mathfrak{p}_{k}\right)$ over $R$ equals $d-k$. In case $\mathcal{S} \diamond(M)=\mathcal{S}(M)$, the ideals $\mathfrak{p}_{k}$ can be chosen to be $z$-ideals.

An ideal $\mathfrak{a}$ of $\mathcal{S}(M)$ is a $z$-ideal if given two continuous semialgebraic functions $f \in \mathfrak{a}$ and $g \in \mathcal{S}(M)$ such that $Z(f) \subset Z(g)$ it holds that $g \in \mathfrak{a}$. Notice that each $z$-ideal is a real ideal. It follows from Theorem 2.6.6 in [2] that if $M$ is locally closed, the $z$-ideals coincide with the radical ideals; in particular, all prime ideals are $z$-ideals. Of course, this is no longer true if $M$ is not locally closed. Even more, it is proved in [12] that classical Łojasiewicz's inequality and Nullstellensatz for $\mathcal{S}(M)$ hold if and only if $M$ is locally closed. In [12] we present versions of such results for $\mathcal{S}^{*}(M)$ when $M$ is an arbitrary semialgebraic set.

In the algebraic case the transcendence degree of the quotient field of the ring of polynomial functions on an irreducible algebraic set $Z \subset C^{m}$ over an algebraically real closed field $C$ coincides with the dimension of $Z$ (see Theorem 11.25 in [1]). The counterpart in the semialgebraic setting is the following.

Theorem 1.3 (Transcendence degrees over $R$ ). Let $\mathfrak{p} \subset \mathcal{S}^{\diamond}(M)$ be a prime ideal. Then,

(i) the transcendence degree of the real closed field $\mathrm{qf}\left(\mathcal{S}^{\diamond}(M) / \mathfrak{p}\right)$ over $R$ is finite and upperly bounded by $\operatorname{dim}(M)$;

(ii) if $\mathfrak{p} \subset \mathcal{S}(M)$ is a prime $z$-ideal, $\mathrm{d}_{M}(\mathfrak{p})=\operatorname{tr} \operatorname{deg}_{R}(\mathrm{qf}(\mathcal{S}(M) / \mathfrak{p}))$.

In the proof of this result semialgebraic pseudo-compactifications play again a main role because for each prime ideal $\mathfrak{p}$ of $\mathcal{S}^{\diamond}(M)$ there exists a 'brimming' semialgebraic pseudo-compactification $X$ of $M$ such that $\operatorname{qf}\left(\mathcal{S}^{\diamond}(M) / \mathfrak{p}\right)=\operatorname{qf}(\mathcal{S}(X) /(\mathfrak{p} \cap$ $\mathcal{S}(X))$ ) (see 3.A.7). 
The article is organized as follows. In Section 2 we provide elementary geometric proofs for the main results in this wok when $M$ is locally closed. In Section 3 we develop the main properties concerning semialgebraic pseudo-compactifications of $M$ and show Theorem 1.3 as a byproduct. Finally, Theorems 1.1 and 1.2 are proved in Section 4.

Acknowledgements. The authors are deeply indebted to the anonymous referees for their careful reading and comments that have substantially improved the exposition and simplified the proof of Theorem 2.1 (iii) in a significant way. The authors are also grateful to S. Schramm for a careful reading of the final version and for the suggestions to refine its writing.

\section{The locally closed case}

In this section we provide an elementary geometric proof of the following compiling result for a locally closed semialgebraic set $M$ using basically Łojasiewicz's inequality and cell decomposition of semialgebraic sets. Recall that a cell is a semialgebraic subset of $R^{m}$, which is semialgebraically homeomorphic to a cube $(0,1)^{d}$.

Theorem 2.1. Let $M \subset R^{m}$ be a locally closed semialgebraic set. Then:

(i) All prime ideals of $\mathcal{S}(M)$ are $z$-ideals.

(ii) For each prime ideal $\mathfrak{p}$ of $\mathcal{S}(M), \mathrm{d}_{M}(\mathfrak{p})+\mathrm{ht}(\mathfrak{p}) \leq \operatorname{dim}(M)$. Thus,

$$
\operatorname{dim}(\mathcal{S}(M)) \leq \operatorname{dim}(M) .
$$

(iii) For each prime ideal $\mathfrak{p}$ of $\mathcal{S}(M)$ the field $\mathrm{qf}(\mathcal{S}(M) / \mathfrak{p})$ is real closed and

$$
\operatorname{tr} \operatorname{deg}_{R}(\mathrm{qf}(\mathcal{S}(M) / \mathfrak{p}))=\mathrm{d}_{M}(\mathfrak{p}) .
$$

Two crucial facts concerning $\mathcal{S}^{\diamond}(M)$ we will use in this work are the following:

- Every closed semialgebraic subset $Z$ of $M$ is the zero set $Z(h)$ of a (bounded with respect to $R$ ) continuous semialgebraic function $h$ on $M$. Take, for instance,

$$
h:=\min \{1, \operatorname{dist}(\cdot, Z)\} \in \mathcal{S}^{*}(M) .
$$

- The restriction homomorphism $\mathcal{S}^{\diamond}(M) \rightarrow \mathcal{S}^{\diamond}(Z),\left.f \mapsto f\right|_{Z}$ is surjective for each closed semialgebraic subset $Z$ of $M$ (see Theorem 3, p. 48, in [9]).

The key to prove Theorem 2.1 (ii) is the following.

Lemma 2.2. Let $\mathfrak{p}, \mathfrak{q}$ be two prime ideals of $\mathcal{S}(M)$ such that $\mathfrak{q} \subsetneq \mathfrak{p}$, where $\mathfrak{q}$ is a $z$-ideal. Then $\mathrm{d}_{M}(\mathfrak{p})<\mathrm{d}_{M}(\mathfrak{q})$.

Proof. Suppose by contradiction that there exist two prime ideals $\mathfrak{p}, \mathfrak{q} \subset \mathcal{S}(M)$ such that $\mathfrak{q} \subsetneq \mathfrak{p}$, where $\mathfrak{q}$ is a $z$-ideal, and $\mathrm{d}_{M}(\mathfrak{p})=\mathrm{d}_{M}(\mathfrak{q})$. Let $g \in \mathfrak{q}$ be such that $\mathrm{d}_{M}(\mathfrak{q})=\operatorname{dim}(Z(g))$. Choose $f \in \mathfrak{p} \backslash \mathfrak{q}$ and define $f^{\prime}:=f^{2}+g^{2}$. Clearly, $f^{\prime} \in \mathfrak{p} \backslash \mathfrak{q}$ and

$$
\operatorname{dim}\left(Z\left(f^{\prime}\right)\right) \leq \operatorname{dim}(Z(g))=\mathrm{d}_{M}(\mathfrak{q})=\mathrm{d}_{M}(\mathfrak{p}) \leq \operatorname{dim}\left(Z\left(f^{\prime}\right)\right)
$$


hence, $\operatorname{dim}\left(Z\left(f^{\prime}\right)\right)=\operatorname{dim}(Z(g))$. Denote $T:=Z(g) \backslash Z\left(f^{\prime}\right)$ and let $h \in \mathcal{S}(M)$ be such that $\mathrm{Cl}_{M}(T)=Z(h)$. We have

$$
Z(g)=\mathrm{Cl}_{M}(T) \cup Z\left(f^{\prime}\right)=Z\left(f^{\prime} h\right) .
$$

Since $g \in \mathfrak{q}$ and $\mathfrak{q}$ is a $z$-ideal, $f^{\prime} h \in \mathfrak{q}$. As $\mathfrak{q}$ is prime and $f^{\prime} \notin \mathfrak{q}$, we deduce $h \in \mathfrak{q} \subset \mathfrak{p}$. Therefore, $h^{\prime}:=h^{2}+{f^{\prime}}^{2} \in \mathfrak{p}$, so $\operatorname{dim}\left(Z\left(h^{\prime}\right)\right) \geq \mathrm{d}_{M}(\mathfrak{p})$. Now

$$
Z\left(h^{\prime}\right)=Z(h) \cap Z\left(f^{\prime}\right) \subset \mathrm{Cl}_{M}(T) \cap(M \backslash T)=\mathrm{Cl}_{M}(T) \backslash T .
$$

By Proposition 2.8.13 in [2] we have

$$
\begin{aligned}
\mathrm{d}_{M}(\mathfrak{p}) \leq \operatorname{dim}\left(Z\left(h^{\prime}\right)\right) & \leq \operatorname{dim}\left(\mathrm{Cl}_{M}(T) \backslash T\right) \\
& <\operatorname{dim}(T) \leq \operatorname{dim}(Z(g))=\mathrm{d}_{M}(\mathfrak{q})=\mathrm{d}_{M}(\mathfrak{p}),
\end{aligned}
$$

which is a contradiction. We conclude $\mathrm{d}_{M}(\mathfrak{p})<\mathrm{d}_{M}(\mathfrak{q})$, as required.

Remark 2.3. Lemma 2.2 is false if $M$ is not locally closed and $\mathfrak{q}$ is not a $z$-ideal. Indeed, consider the triangle $M:=\left\{(x, y) \in R^{2}: 0<y \leq x \leq 1\right\} \cup\{p:=(0,0)\}$ and the prime ideal $\mathfrak{q}$ of all semialgebraic functions $f \in \mathcal{S}(M)$ that extend continuously by 0 to $M \cup((0, \varepsilon] \times\{0\})$ for some $\varepsilon>0$ (depending on $f$ ). Clearly, $\mathfrak{q} \subsetneq \mathfrak{m}_{p}$ and a straightforward computation shows that $\mathrm{d}_{M}(\mathfrak{q})=\mathrm{d}_{M}\left(\mathfrak{m}_{p}\right)=0$.

Proof of statements (i) and (ii) in Theorem 2.1. (i) As the semialgebraic set $M$ is locally closed, the prime ideals of $\mathcal{S}(M)$ are $z$-ideals by Łojasiewicz's inequality (Theorem 2.6.6 in [2]).

(ii) Let us denote $d:=\operatorname{dim}(M)$. Given a chain of prime ideals $\mathfrak{p}_{0} \subsetneq \cdots \subsetneq \mathfrak{p}_{r}$ in $\mathcal{S}(M)$, we get by Lemma 2.2 that $\mathrm{d}_{M}\left(\mathfrak{p}_{r}\right)<\cdots<\mathrm{d}_{M}\left(\mathfrak{p}_{0}\right) \leq d$, and $\operatorname{sod}_{M}\left(\mathfrak{p}_{r}\right) \leq$ $d-r$. Thus, $\operatorname{ht}(\mathfrak{p})+\mathrm{d}_{M}(\mathfrak{p}) \leq d$ for every prime ideal $\mathfrak{p} \subset \mathcal{S}(M)$, as required.

\section{A. Proof of Theorem 2.1 (iii)}

A standard tool when dealing with prime $z$-ideals is its ultrafilter description [3], [4]. The prime $z$-ideals of $\mathcal{S}(M)$ are in natural bijection with the ultrafilters of semialgebraic subsets of $R^{m}$ containing $M$. The ultrafilter $\mathcal{U}_{\mathfrak{p}}$ associated with a prime $z$-ideal $\mathfrak{p}$ of $\mathcal{S}(M)$ admits a description in terms of $\mathrm{d}:=\mathrm{d}_{M}(\mathfrak{p})$ : a semialgebraic set $S \subset R^{m}$ belongs to $\mathcal{U}_{\mathfrak{p}}$ if and only if there exists $f \in \mathfrak{p}$ such that $\operatorname{dim}(Z(f) \backslash S)<\mathrm{d}$. This ultrafilter provides a maximal ideal of the ring $\mathcal{D}(M)$ of (not necessarily continuous) semialgebraic functions on $M$ :

$$
\begin{aligned}
\widetilde{\mathfrak{p}} & :=\left\{g \in \mathcal{D}(M): Z(g) \in \mathcal{U}_{\mathfrak{p}}\right\} \\
& =\{g \in \mathcal{D}(M): \exists f \in \mathfrak{p} \text { such that } \operatorname{dim}(Z(f) \backslash Z(g))<\mathrm{d}\} .
\end{aligned}
$$

2.A.1. As $\mathcal{U}_{\mathfrak{p}}$ is an ultrafilter, it holds that $\widetilde{\mathfrak{p}}$ is a maximal ideal. However, an easy direct proof of this fact follows from the definition of $\widetilde{p}$. 
To prove that $\widetilde{\mathfrak{p}}$ is an ideal, only its closedness with respect to addition requires a comment. If $g_{1}, g_{2} \in \widetilde{\mathfrak{p}}$ and $f_{1}, f_{2} \in \mathfrak{p}$ satisfy $\operatorname{dim}\left(Z\left(f_{i}\right) \backslash Z\left(g_{i}\right)\right)<\mathrm{d}$, then $f_{1}^{2}+f_{2}^{2} \in \mathfrak{p}$, and

$$
\begin{aligned}
Z\left(f_{1}^{2}+f_{2}^{2}\right) \backslash Z\left(g_{1}+g_{2}\right) \subset Z\left(f_{1}\right) \cap Z\left(f_{2}\right) \backslash & \left(Z\left(g_{1}\right) \cap Z\left(g_{2}\right)\right) \\
& \subset\left(Z\left(f_{1}\right) \backslash Z\left(g_{1}\right)\right) \cup\left(Z\left(f_{2}\right) \backslash Z\left(g_{2}\right)\right) .
\end{aligned}
$$

Consequently, the dimension of $Z\left(f_{1}^{2}+f_{2}^{2}\right) \backslash Z\left(g_{1}+g_{2}\right)$ is strictly smaller than d, so $g_{1}+g_{2} \in \mathfrak{p}$.

To check that $\widetilde{\mathfrak{p}}$ is a maximal ideal, we show that $\mathcal{D}(M) / \widetilde{\mathfrak{p}}$ is a field. Indeed, if $f \in \mathcal{D}(M) \backslash \widetilde{\mathfrak{p}}$, consider $g \in \mathcal{D}(M)$ that takes values 1 on $Z(f)$ and 0 on $M \backslash Z(f)$. As $f g=0 \in \widetilde{\mathfrak{p}}$ and $f \notin \widetilde{\mathfrak{p}}$, we have $g \in \widetilde{\mathfrak{p}}$. As $Z(f+g)=\varnothing$, it holds $\frac{1}{f+g} \in \mathcal{D}(M)$, so $f+\widetilde{\mathfrak{p}}$ is a unit in $\mathcal{D}(M) / \widetilde{\mathfrak{p}}$.

2.A.2. Each $f \in \mathcal{D}(M)$ is determined modulo $\widetilde{\mathfrak{p}}$ by its restriction to any $S \in \mathcal{U}_{\mathfrak{p}}$.

2.A.3. Given $f \in \mathcal{D}(M) \backslash \widetilde{\mathfrak{p}}$, there exists a cell $C \in \mathcal{U}_{\mathfrak{p}}$ of dimension d such that $\left.f\right|_{C}$ is continuous (even Nash) and never vanishes on $C$.

Let $S \in \mathcal{U}_{\mathfrak{p}}$ be of dimension d. Let $\mathcal{C}:=\left\{C_{1}, \ldots, C_{s}\right\}$ be a cell decomposition of $S$ such that $\left.f\right|_{C_{i}}$ is continuous (even Nash) and either $\left.f\right|_{C_{i}}=0$ or $Z(f) \cap C_{i}=\varnothing$ (use Proposition 2.9.10 and Definition 9.1.11 in [2]). Assume $C:=C_{1} \in \mathcal{U}_{\mathfrak{p}}$. Clearly, $\operatorname{dim}(C)=\mathrm{d}$ and as $f \notin \widetilde{\mathfrak{p}}$, it holds $Z(f) \cap C=\varnothing$.

2.A.4. As $\widetilde{\mathfrak{p}} \cap \mathcal{S}(M)=\mathfrak{p}$, we have $\mathcal{S}(M) / \mathfrak{p} \hookrightarrow \mathcal{D}(M) / \widetilde{\mathfrak{p}}$. In fact, $\mathcal{D}(M) / \widetilde{\mathfrak{p}} \cong$ $\mathrm{qf}(\mathcal{S}(M) / \mathfrak{p})$. It is enough to show that given $f \in \mathcal{D}(M) \backslash \widetilde{\mathfrak{p}}$, there exist $g_{1}, f_{1} \in$ $\mathcal{S}(M) \backslash \widetilde{\mathfrak{p}}$ such that $g_{1} f-f_{1} \in \widetilde{\mathfrak{p}}$.

Let $C \in \mathcal{U}_{\mathfrak{p}}$ be a cell of dimension d such that $\left.f\right|_{C}$ is continuous and $Z(f) \cap C$ $=\varnothing$. As $C$ is locally closed, $\mathrm{Cl}_{R^{m}}(C) \backslash C$ is a closed subset of $R^{m}$ and there exists $g \in \mathcal{S}\left(R^{m}\right)$ such that $Z(g)=\mathrm{Cl}_{R^{m}}(C) \backslash C$; clearly, $g \notin \mathfrak{p}$. By Theorem 2.6.6 in [2], there exists an integer $k \geq 1$ such that $g^{k} f \in \mathcal{S}\left(\mathrm{Cl}_{R^{m}}(C)\right)$. As $\mathrm{Cl}_{M}(C)$ is closed in $M$, there exists $f_{1} \in \mathcal{S}(M)$ such that $\left.f_{1}\right|_{\mathrm{Cl}_{M}(C)}=\left.\left(g^{k} f\right)\right|_{\mathrm{Cl}_{M}(C)}$. As $C \subset$ $Z\left(f_{1}-g^{k} f\right)$, it holds $f_{1}-g^{k} f \in \widetilde{\mathfrak{p}}$, as wanted.

2.A.5. Some of the previous arguments become straightforward if one notes that $\mathcal{D}(M)$ is the von Neumann regularization of $\mathcal{S}(M)$. However, as their proofs are quite short and elementary we have included them for the sake of the reader.

2.A.6. It holds that $\operatorname{tr} \operatorname{deg}_{R}(\mathcal{D}(M) / \widetilde{\mathfrak{p}})=\mathrm{d}$.

Let $C \in \mathcal{U}_{\mathfrak{p}}$ be a cell of dimension d. We may assume by Proposition 2.9.10 in [2] that there exists a Nash diffeomorphism $\mathrm{h}:=\left(h_{1}, \ldots, h_{\mathrm{d}}\right): C \rightarrow(0,1)^{\mathrm{d}}$. Pick $f_{i} \in \mathcal{D}(M)$ such that $\left.f_{i}\right|_{C}=h_{i}$.

We claim: the $R$-homomorphism

$$
R\left[\mathrm{x}_{1}, \ldots, \mathrm{x}_{\mathrm{d}}\right] \rightarrow \mathcal{D}(M) / \widetilde{\mathfrak{p}}, P \mapsto P\left(f_{1}, \ldots, f_{\mathrm{d}}\right)+\widetilde{\mathfrak{p}}
$$

is injective. Consequently, $\operatorname{tr} \operatorname{deg}_{R}(\mathcal{D}(M) / \widetilde{\mathfrak{p}}) \geq \mathrm{d}$. 
Indeed, if $P\left(f_{1}, \ldots, f_{\mathrm{d}}\right) \in \widetilde{\mathfrak{p}}$, there exists $g_{2} \in \mathfrak{p}$ such that

$$
\operatorname{dim}\left(Z\left(g_{2}\right) \backslash Z\left(P\left(f_{1}, \ldots, f_{\mathrm{d}}\right)\right)\right)<\mathrm{d}
$$

and $Z\left(g_{2}\right) \subset \mathrm{Cl}_{M}(C)$. Thus, $P$ vanishes on a non-empty open subset of $(0,1)^{\mathrm{d}}$, so $P=0$.

Next, if $f \in \mathcal{D}(M)$, we may assume that $\left.f\right|_{C}$ is a Nash function, so there exists a non-zero polynomial $Q \in R\left[\mathrm{x}_{1}, \ldots, \mathrm{x}_{\mathrm{d}}, \mathrm{y}\right]$ such that $Q\left(f_{1}, \ldots, f_{\mathrm{d}}, f\right) \in \widetilde{\mathfrak{p}}$. Hence, $\operatorname{tr} \operatorname{deg}_{R}(\mathcal{D}(M) / \widetilde{\mathfrak{p}})=\mathrm{d}$.

2.A.7. $\mathcal{D}(M) / \widetilde{\mathfrak{p}}$ is a real closed field. As the field $\mathcal{D}(M) / \widetilde{\mathfrak{p}}$ admits a unique ordering (because $\left.\left(f-(\sqrt{|f|})^{2}\right)\left(f+(\sqrt{|f|})^{2}\right)=0 \in \widetilde{\mathfrak{p}}\right)$, it is enough to prove the following: each monic polynomial $F:=\mathrm{y}^{2 n+1}+\sum_{k=0}^{2 n}\left(a_{k}+\widetilde{\mathfrak{p}}\right) \mathrm{y}^{k} \in(\mathcal{D}(M) / \widetilde{\mathfrak{p}})[\mathrm{y}]$ of odd degree has a root in $\mathcal{D}(M) / \widetilde{\mathfrak{p}}$.

Consider the polynomial

$$
P(\mathrm{a}, \mathrm{y})=\mathrm{y}^{2 n+1}+\sum_{k=0}^{2 n} \mathrm{a}_{k} \mathrm{y}^{k} \in R[\mathrm{a}, \mathrm{y}] .
$$

By Theorem 2.3.1 in [2], there exists a partition of $R^{2 n+1}$ into finitely many semialgebraic sets $A_{1}, \ldots, A_{\ell}$ and finitely many continuous semialgebraic functions $\zeta_{i j}: A_{i} \rightarrow R$ such that the roots of $P(u, \mathrm{y})$ for each $u \in A_{i}$ are $\zeta_{i j}(u)$. Consider the map $a: M \rightarrow R^{2 n+1}, x \mapsto\left(a_{k}(x)\right)_{k}$ and the finite semialgebraic partition $\left\{a^{-1}\left(A_{i}\right)\right\}_{i}$ of $M$. Assume $a^{-1}\left(A_{1}\right) \in \mathcal{U}_{\mathfrak{p}}$, so $\zeta_{11} \circ a$ is a root of $F$ on $a^{-1}\left(A_{1}\right)$ and we are done.

\section{Semialgebraic pseudo-compactifications}

A semialgebraic pseudo-compactification of $M$ is a pair $(X, j)$ constituted of a closed and bounded semialgebraic set $X \subset R^{n}$ and a semialgebraic embedding $\mathrm{j}: M \hookrightarrow X$ whose image is dense in $X$. It holds that $\mathcal{S}(X)=\mathcal{S}^{*}(X)$ because the image of a bounded and closed semialgebraic set under a continuous semialgebraic function is again bounded and closed. It is well-known that a bounded and closed subset of $R^{n}$ is compact only if $R=\mathbb{R}$. The embedding $\mathrm{j}$ induces an $R$-monomorphism $\mathrm{j}^{\diamond}: \mathcal{S}(X) \hookrightarrow \mathcal{S}^{\diamond}(M), f \mapsto f \circ \mathrm{j}$ and we will denote $\mathfrak{a} \cap \mathcal{S}(X):=$ $\left(j^{\diamond}\right)^{-1}(\mathfrak{a})$ for every ideal $\mathfrak{a}$ of $\mathcal{S}^{\diamond}(M)$. Sometimes it will be useful to assume that the semialgebraic set $M$ is bounded. Namely, the semialgebraic homeomorphism between the open ball $\mathcal{B}_{m} \subset R^{m}$ of center 0 and radius 1 and $R^{m}$,

$$
\mathrm{h}: \mathcal{B}_{m} \rightarrow R^{m} x \mapsto \frac{x}{\sqrt{1-\|x\|^{2}}}
$$

induces an $R$-isomorphism $\mathcal{S}^{\diamond}(M) \rightarrow \mathcal{S}^{\diamond}\left(\mathrm{h}^{-1}(M)\right), f \mapsto f \circ \mathrm{h}$. Thus, we may always assume that $M$ is bounded and in particular that the closure $\mathrm{Cl}(M)$ of $M$ (in $R^{m}$ ) is a semialgebraic pseudo-compactification of $M$. 


\section{A. Properties of the semialgebraic pseudo-compactifications}

The following properties are essential:

3.A.1. For each finite family $\mathcal{F}:=\left\{f_{1}, \ldots, f_{r}\right\} \subset \mathcal{S}^{*}(M)$ there exist a semialgebraic pseudo-compactification $\left(X_{\mathcal{F}}, j_{\mathcal{F}}\right)$ of $M$ and $F_{1}, \ldots, F_{r} \in \mathcal{S}\left(X_{\mathcal{F}}\right)$ such that $f_{i}=F_{i} \circ j \mathcal{F}$.

Assume that $M$ is bounded and consider $X_{\mathcal{F}}:=\mathrm{Cl}\left(\operatorname{graph}\left(f_{1}, \ldots, f_{r}\right)\right)$,

$$
\mathrm{j}_{\mathcal{F}}: M \hookrightarrow X_{\mathcal{F}}, x \mapsto\left(x, f_{1}(x), \ldots, f_{r}(x)\right)
$$

and $F_{i}:=\left.\pi_{m+i}\right|_{X_{\mathcal{F}}}$ where

$$
\pi_{m+i}: R^{m+r} \rightarrow R, x:=\left(x_{1}, \ldots, x_{m+r}\right) \mapsto x_{m+i} \quad \text { for } i=1, \ldots, r .
$$

3.A.2. Given a chain of prime ideals $\mathfrak{p}_{0} \subsetneq \cdots \subsetneq \mathfrak{p}_{r}$ of $\mathcal{S}^{*}(M)$, there exists a semialgebraic pseudo-compactification $(X, j)$ of $M$ such that the prime ideals $\mathfrak{q}_{i}:=$ $\mathfrak{p}_{i} \cap \mathcal{S}(X)$ constitute a chain $\mathfrak{q}_{0} \subsetneq \cdots \subsetneq \mathfrak{q}_{r}$ in $\mathcal{S}(X)$.

It is enough to pick $f_{i} \in \mathfrak{p}_{i} \backslash \mathfrak{p}_{i-1}$ for $1 \leq i \leq r$ and to consider the semialgebraic pseudo-compactification of $M$ provided for the family $\mathcal{F}:=\left\{f_{1}, \ldots, f_{r}\right\}$ by 3.A.1.

3.A.3. Let $\mathfrak{F}_{M}$ be the collection of all semialgebraic pseudo-compactifications of $M$. Given $\left(X_{1}, j_{1}\right),\left(X_{2}, j_{2}\right) \in \mathfrak{F}_{M}$, we say $\left(X_{1}, j_{1}\right) \preccurlyeq\left(X_{2}, j_{2}\right)$ if there exists a (unique) continuous (surjective) semialgebraic map $\rho:=\rho_{X_{1}, X_{2}}: X_{2} \rightarrow X_{1}$ such that $\rho \circ \mathrm{j}_{2}=\mathrm{j}_{1}$. The uniqueness of $\rho$ follows because $\mathrm{j}_{i}(M)$ is dense in $X_{i}$ and $\left.\rho\right|_{j_{2}(M)}=\mathrm{j}_{1} \circ \mathrm{j}_{2}^{-1}$ if we understand $\mathrm{j}_{2}$ as the homeomorphism $M \rightarrow \mathrm{j}_{2}(M)$. We claim: $\left(\mathfrak{F}_{M}, \preccurlyeq\right)$ is an up-directed set.

Let $\left(X_{1}, j_{1}\right),\left(X_{2}, j_{2}\right) \in \mathfrak{F}_{M}$ and consider the continuous semialgebraic map

$$
\mathrm{j}_{3}: M \rightarrow X_{3}:=\mathrm{Cl}\left(\left(\mathrm{j}_{1}, \mathrm{j}_{2}\right)(M)\right), x \mapsto\left(\mathrm{j}_{1}(x), \mathrm{j}_{2}(x)\right) .
$$

Notice that $\left(X_{3}, j_{3}\right) \in \mathfrak{F}_{M}$ and $\left(X_{1}, j_{1}\right) \preccurlyeq\left(X_{3}, j_{3}\right)$ and $\left(X_{2}, j_{2}\right) \preccurlyeq\left(X_{3}, j_{3}\right)$.

3.A.4. We have a collection of rings $\{\mathcal{S}(X)\}_{(X, j) \in \mathfrak{F}_{M}}$ and $R$-monomorphisms

$$
\rho_{X_{1}, X_{2}}^{*}: \mathcal{S}\left(X_{1}\right) \rightarrow \mathcal{S}\left(X_{2}\right), f \mapsto f \circ \rho_{X_{1}, X_{2}}
$$

for $\left(X_{1}, j_{1}\right) \preccurlyeq\left(X_{2}, j_{2}\right)$ such that

- $\rho_{X_{1}, X_{1}}^{*}=\mathrm{id}$, and

- $\rho_{X_{1}, X_{3}}^{*}=\rho_{X_{2}, X_{3}}^{*} \circ \rho_{X_{1}, X_{2}}^{*}$ if $\left(X_{1}, j_{1}\right) \preccurlyeq\left(X_{2}, j_{2}\right) \preccurlyeq\left(X_{3}, j_{3}\right)$.

We conclude: the ring $\mathcal{S}^{*}(M)$ is the direct limit of the up-directed system $\left\langle\mathcal{S}(X), \rho_{X_{1}, X_{2}}^{*}\right\rangle$ together with the $R$-homomorphisms $j^{*}: \mathcal{S}(X) \hookrightarrow \mathcal{S}^{*}(M)$, where $(X, \mathrm{j}) \in \mathfrak{F}_{M}$. 
3.A.5. On the other hand, the ring $\mathcal{S}(M)$ is the localization $\mathcal{S}^{*}(M)_{\mathcal{W}(M)}$ of $\mathcal{S}^{*}(M)$ at the multiplicative set $\mathcal{W}(M)$ of those functions $f \in \mathcal{S}^{*}(M)$ such that $Z(f)=\varnothing$. In particular, if $\mathfrak{p}$ is a prime ideal of $\mathcal{S}^{*}(M)$ that does not meet $\mathcal{W}(M)$, then $\mathrm{qf}\left(\mathcal{S}^{*}(M) / \mathfrak{p}\right)=\mathrm{qf}(\mathcal{S}(M) / \mathfrak{p} \mathcal{S}(M))$.

This is pretty evident since each function $f \in \mathcal{S}(M)$ is the quotient $f=g / h$, where $g:=\frac{f}{1+|f|} \in \mathcal{S}^{*}(M)$ and $h:=\frac{1}{1+|f|} \in \mathcal{W}(M)$.

3.A.6. We conclude from 3.A.2 and 3.A.5 that

$$
\operatorname{dim}(\mathcal{S}(M)) \leq \operatorname{dim}\left(\mathcal{S}^{*}(M)\right) \leq \sup _{(X, \mathrm{j}) \in \mathfrak{F}_{M}}\{\operatorname{dim}(\mathcal{S}(X))\}
$$

3.A.7. Let $\mathfrak{p}$ be a prime ideal of $\mathcal{S}^{\diamond}(M)$. Then there exists a semialgebraic pseudocompactification $(X, \mathrm{j})$ of $M$ such that

$$
\operatorname{qf}(\mathcal{S}(X) /(\mathfrak{p} \cap \mathcal{S}(X)))=\operatorname{qf}\left(\mathcal{S}^{\diamond}(M) / \mathfrak{p}\right) .
$$

We refer to $(X, \mathrm{j})$ as a brimming semialgebraic pseudo-compactification of $M$ for $\mathfrak{p}$. In particular, $\mathrm{qf}\left(\mathcal{S}^{\diamond}(M) / \mathfrak{p}\right)$ is a real closed field.

Proof. By 3.A.5 it is enough to consider the case $\mathcal{S}^{\diamond}(M)=\mathcal{S}^{*}(M)$ and we assume moreover that $M$ is bounded. Consider the field $F:=\operatorname{qf}\left(\mathcal{S}^{*}(M) / \mathfrak{p}\right)$ and the $R$ homomorphism

$$
\varphi: \mathcal{S}^{*}(M) \rightarrow \mathcal{S}^{*}(M) / \mathfrak{p} \hookrightarrow F
$$

For each finite set $\mathcal{F}:=\left\{f_{1}, \ldots, f_{r}\right\} \subset \mathcal{S}^{*}(M)$ consider the semialgebraic pseudocompactification $\left(X_{\mathcal{F}}, j_{\mathcal{F}}\right)$ constructed in 3.A.1 and define $\mathfrak{p}_{\mathcal{F}}:=\mathfrak{p} \cap \mathcal{S}\left(X_{\mathcal{F}}\right)$. Fix a finite subset $\mathcal{F}_{0}$ of $\mathcal{S}^{*}(M)$ such that

$$
\left.\mathrm{d}_{X_{\mathcal{F}_{0}}}\left(\mathfrak{p}_{\mathcal{F}_{0}}\right)=\max _{\mathcal{F}}\left\{\mathrm{d}_{X_{\mathcal{F}}}\left(\mathfrak{p}_{\mathcal{F}}\right)\right)\right\}
$$

where $\mathcal{F}$ runs over all finite subsets of $\mathcal{S}^{*}(M)$ and denote $X_{0}:=X_{\mathcal{F}_{0}}$. Clearly, $F_{0}:=\operatorname{qf}\left(\mathcal{S}\left(X_{0}\right) /\left(\mathfrak{p} \cap \mathcal{S}\left(X_{0}\right)\right)\right) \subset F$. Next, fix $f \in \mathcal{S}^{*}(M) \backslash \mathfrak{p}$ and consider the set $\mathcal{F}_{1}:=\mathcal{F}_{0} \cup\{f\}$ and the semialgebraic pseudo-compactification $X_{1}:=X_{\mathcal{F}_{1}}$ of $M$. The projection onto all coordinates except the last one induces a surjective semialgebraic map $\rho: X_{1} \rightarrow X_{0}$ whose restriction to $M$ is a semialgebraic homeomorphism. This map induces the $R$-monomorphism $\mathcal{S}\left(X_{0}\right) \hookrightarrow \mathcal{S}\left(X_{1}\right), h \mapsto h \circ \rho$. We have the following commutative diagrams:
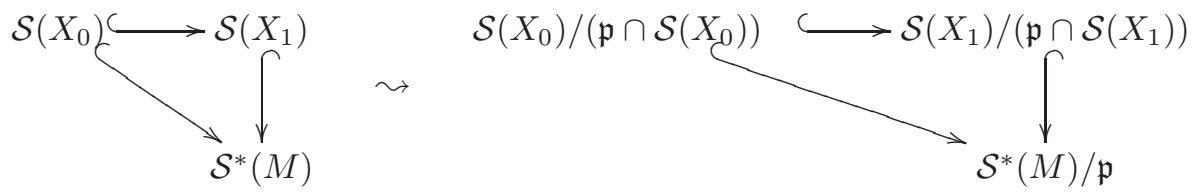

so $F_{0} \subset F_{1}:=\operatorname{qf}\left(\mathcal{S}\left(X_{1}\right) /\left(\mathfrak{p} \cap \mathcal{S}\left(X_{1}\right)\right)\right) \subset F$. As $f$ admits an extension $\widehat{f}$ to $X_{1}$, to see that $f+\mathfrak{p}$ is algebraic over $F_{0}$ it is enough to prove that $\widehat{f}+\left(\mathfrak{p} \cap \mathcal{S}\left(X_{1}\right)\right)$ is algebraic over $F_{0}$. 
For this it is sufficient to show that the transcendence degrees of $F_{0}$ and $F_{1}$ over $R$ coincide. Since $F_{0} \subset F_{1}$, it follows, from Theorem 2.1 (iii) and equation (3.1), that

$$
\begin{aligned}
\operatorname{tr} \operatorname{deg}_{R}\left(F_{0}\right) \leq \operatorname{tr} \operatorname{deg}_{R}\left(F_{1}\right) & =\mathrm{d}_{X_{1}}\left(\mathfrak{p}_{\mathcal{F}_{1}}\right) \\
& \left.\leq \max _{\mathcal{F}}\left\{\mathrm{d}_{X_{\mathcal{F}}}\left(\mathfrak{p}_{\mathcal{F}}\right)\right)\right\}=\mathrm{d}_{X_{0}}\left(\mathfrak{p}_{\mathcal{F}_{0}}\right)=\operatorname{tr} \operatorname{deg}_{R}\left(F_{0}\right)
\end{aligned}
$$

As both $F_{0}$ and $F_{1}$ are real closed fields, we conclude that $F_{0}=F_{1}$, so $f+\mathfrak{p} \in F_{0}$ and $F=F_{0}$.

Remark 3.1. Observe that not all semialgebraic pseudo-compactifications $X$ of $M$ are in general brimming semialgebraic pseudo-compactifications for a given prime ideal $\mathfrak{p}$ of $\mathcal{S}^{*}(M)$. Indeed, consider the triangle $M:=\left\{(x, y) \in R^{2}: 0<y \leq\right.$ $x \leq 1\}$ and the prime ideal $\mathfrak{p}$ of all semialgebraic functions $f \in \mathcal{S}^{*}(M)$ that extend continuously by 0 to $M \cup((0, \varepsilon] \times\{0\})$ for some $\varepsilon>0$ (depending on $f$ ). As one can check, $\mathrm{qf}\left(\mathcal{S}^{*}(M) / \mathfrak{p}\right)$ is isomorphic to the field $R\left(\left(\mathrm{t}^{*}\right)\right)$ alg of algebraic Puiseux series with coefficients in $R$. Let $(X, j)$ be the (semialgebraic) Alexandroff's pseudocompactification of $M$ and denote $\{\infty\}:=X \backslash j(M)$. It holds $\mathfrak{p} \cap \mathcal{S}(X)=\mathfrak{m}_{\infty}^{*}$, so $\mathcal{S}(X) /(\mathfrak{p} \cap \mathcal{S}(X)) \cong R$.

\section{B. Proof of Theorem 1.3}

Now we are ready to prove Theorem 1.3. Assertion (i) follows readily from Theorem 2.1 (iii) and paragraph 3.A.7. In order to prove (ii), pick a brimming semialgebraic pseudo-compactification $(X, \mathrm{j})$ of $M$ for $\mathfrak{p}$. By Theorem 2.1 (iii),

$$
\mathrm{d}_{X}(\mathfrak{p} \cap \mathcal{S}(X))=\operatorname{tr} \operatorname{deg}_{R}(\operatorname{qf}(\mathcal{S}(X) /(\mathfrak{p} \cap \mathcal{S}(X))))=\operatorname{tr} \operatorname{deg}_{R}(\operatorname{qf}(\mathcal{S}(M) / \mathfrak{p})) .
$$

It only remains to check $\mathrm{d}_{X}(\mathfrak{p} \cap \mathcal{S}(X))=\mathrm{d}_{M}(\mathfrak{p})$. The inequality $\mathrm{d}_{X}(\mathfrak{p} \cap \mathcal{S}(X)) \geq$ $\mathrm{d}_{M}(\mathfrak{p})$ is clear. For the converse inequality, let $f \in \mathfrak{p}$ be such that $\mathrm{d}_{M}(\mathfrak{p})=$ $\operatorname{dim}(Z(f))$ and pick a function $g \in \mathcal{S}(X)$ such that $\mathrm{Cl}_{X}(Z(f))=Z(g)$. As $\mathfrak{p}$ is a $z$-ideal, $g \in \mathfrak{p}$, so $g \in \mathfrak{p} \cap \mathcal{S}(X)$. Therefore,

$$
\mathrm{d}_{M}(\mathfrak{p})=\operatorname{dim}(Z(f))=\operatorname{dim}(Z(g)) \geq \mathrm{d}_{X}(\mathfrak{p} \cap \mathcal{S}(X)),
$$

as required.

\section{Krull dimension of rings of continuous semialgebraic func- tions}

In this section we prove Theorems 1.1 and 1.2 for a general semialgebraic set $M$. We begin with the following key example, which is very close to Lemma 8.8 in [8], although the latter is focused on the real spectrum of a polynomial ring. We denote the relative interior of a simplex $\sigma$ with $\sigma^{0}$. 
Example 4.1. (i) Let $X:=[0,1]^{n}$ and define $\mathfrak{p}$ as the set of all continuous semialgebraic functions $f \in \mathcal{S}(X)$ satisfying the following: for each semialgebraic triangulation $(K, \Phi)$ of $X$ compatible with $Z(f)$ it holds $\Phi(\sigma) \subset Z(f)$ where

(4.1.1) $\sigma \in K$ is an $n$-dimensional simplex such that for each $d=0, \ldots, n$ there exists a d-dimensional face $\tau_{d}$ of $\sigma$ such that $\Phi\left(\tau_{d}\right) \subset\left\{\mathrm{x}_{d+1}=0, \ldots, \mathrm{x}_{n}=0\right\}$.

Using the straightforward property (4.1.2) stated below, one shows that $\sigma$ is uniquely determined by (4.1.1). We call $\sigma$ the indicator simplex for $(K, \Phi)$.

(4.1.2) Let $\tau \subset R^{d}$ be a simplex of dimension $d$ and $\eta_{1}, \eta_{2}$ be two simplices contained in $R^{d} \times[0, \infty)$ that have $\tau$ as a common face. Then $\eta_{1}^{0} \cap \eta_{2}^{0} \neq \varnothing$.

(4.1.3) It holds that $\mathfrak{p}$ is a prime ideal of $\mathcal{S}(X)$ and as $\operatorname{dim}(\sigma)=n$, it is clear that $\mathrm{d}_{X}(\mathfrak{p})=n$.

Only the primality of $\mathfrak{p}$ requires a comment. Indeed, let $f_{1}, f_{2} \in \mathcal{S}(X)$ be such that $f_{1} f_{2} \in \mathfrak{p}$ and $(K, \Phi)$ be a semialgebraic triangulation of $X$ compatible with $Z\left(f_{1}\right)$ and $Z\left(f_{2}\right)$. Let $\sigma$ be an indicator simplex for $(K, \Phi)$. Since $\Phi(\sigma) \subset$ $Z\left(f_{1} f_{2}\right)$ and $(K, \Phi)$ is compatible with $Z\left(f_{i}\right)$, we may assume $\Phi\left(\sigma^{0}\right) \subset Z\left(f_{1}\right)$; hence, $\Phi(\sigma) \subset Z\left(f_{1}\right)$. Thus, $f_{1} \in \mathfrak{p}$, so $\mathfrak{p}$ is a prime ideal.

(ii) Write $X_{n}:=[0,1]^{n}$. We claim: there is a chain of prime ideals $\mathfrak{q}_{0} \subsetneq \cdots \subsetneq$ $\mathfrak{q}_{n}:=\mathfrak{m}_{0}$ in $\mathcal{S}\left(X_{n}\right)$ such that $\mathrm{d}_{X_{n}}\left(\mathfrak{q}_{k}\right)=n-k$ for $k=0, \ldots, n$.

For each $k=1, \ldots, n$ define $X_{k}:=[0,1]^{k} \times\{0\}^{n-k} \subset R^{n}$. Clearly, $\{0\} \subsetneq X_{1} \subsetneq$ $\cdots \subsetneq X_{n}$ is a chain of closed subsets of $X_{n}$. The restriction $R$-homomorphism $\varphi_{k}$ : $\mathcal{S}\left(X_{n}\right) \rightarrow \mathcal{S}\left(X_{k}\right),\left.f \mapsto f\right|_{X_{k}}$ is surjective, so the prime ideal $\mathfrak{p}_{k}$ constructed for $X_{k}$ in (i) provides a prime ideal $\mathfrak{q}_{n-k}:=\varphi_{k}^{-1}\left(\mathfrak{p}_{k}\right)$ such that $\mathrm{d}_{X_{n}}\left(\mathfrak{q}_{n-k}\right)=\mathrm{d}_{X_{k}}\left(\mathfrak{p}_{k}\right)=k$. In addition it holds $\mathfrak{q}_{0} \subsetneq \ldots \subsetneq \mathfrak{q}_{n}:=\mathfrak{m}_{0}$.

\section{A. Proof of the main results}

We are ready to address the proofs of Theorems 1.1 and 1.2.

Proof of Theorem 1.1. Combining Theorem 2.1 (ii) and 3.A.6, it holds

$$
\operatorname{dim}(\mathcal{S}(M)) \leq \operatorname{dim}\left(\mathcal{S}^{*}(M)\right) \leq \sup _{(X, \mathrm{j}) \in \mathfrak{F}_{M}}\{\operatorname{dim}(\mathcal{S}(X))\}=\operatorname{dim}(M) .
$$

It remains to show $\operatorname{dim}(M) \leq \operatorname{dim}(\mathcal{S}(M))$. This follows from Theorem 1.2, which we prove next.

Proof of Theorem 1.2. Let $\mathcal{B}$ be a closed ball centered in $p$ such that $\operatorname{dim}_{p}(M)=$ $\operatorname{dim}(M \cap \mathcal{B})$. The $R$-homomorphism $\varphi: \mathcal{S}(M) \rightarrow \mathcal{S}(M \cap \mathcal{B})$ is surjective and $\operatorname{ker} \varphi$ is contained in each prime ideal $\mathfrak{p} \subset \mathfrak{m}_{p}$, so ht $\operatorname{SoM}_{(M)}\left(\mathfrak{m}_{p}\right)=\operatorname{ht}_{\mathcal{S}(M \cap \mathcal{B})}\left(\varphi\left(\mathfrak{m}_{p}\right)\right)$. Thus, substituting $M$ by $M \cap \mathcal{B}$, we may assume $\operatorname{dim}_{p}(M)=\operatorname{dim}(M)$.

4.A.1. There is a semialgebraic embedding $\mathrm{h}:[0,1]^{d} \hookrightarrow M$ such that $\mathrm{h}(0)=p$.

Indeed, let $\mathcal{C}:=\left\{C_{1}, \ldots, C_{s}\right\}$ be a (finite) semialgebraic cellular decomposition of $M$ compatible with $\{p\}$ (see Definition 9.1.11 and Proposition 9.1.12 [2]). Without loss of generality suppose $p \in \mathrm{Cl}_{M}\left(C_{1}\right), C_{1} \subset M$, $\operatorname{dim}\left(C_{1}\right)=d:=\operatorname{dim}_{p}(M)$. 
Denote the characteristic map of the cell $C_{1}$ by g: $[0,1]^{d} \rightarrow X$. The restriction $\left.\mathrm{g}\right|_{(0,1)^{d}}:(0,1)^{d} \rightarrow C_{1}$ is a semialgebraic homeomorphism onto $C_{1}$ and we may assume $\mathrm{g}(0)=p$. Consider the $d$-dimensional bounded and closed affine parallelepiped

$$
T_{0}:=\left\{\sum_{i=1}^{d} \lambda_{i} \mathrm{p}_{i}: 0 \leq \lambda_{i} \leq 1\right\} \subset\{0\} \cup(0,1)^{d}
$$

generated by the origin and the points $\mathrm{p}_{i}:=\frac{1}{d+2}\left(1, \ldots, 1,2^{(i)}, 1, \ldots, 1\right)$ for $i=$ $1, \ldots, d$. Of course,

$$
\mathrm{h}_{0}:[0,1]^{d} \rightarrow T_{0}, \lambda:=\left(\lambda_{1}, \ldots, \lambda_{d}\right) \mapsto \sum_{i=1}^{d} \lambda_{i} \mathrm{p}_{i}
$$

is a semialgebraic homeomorphism and satisfies $\mathrm{h}_{0}(0)=0$. The semialgebraic map $\mathrm{h}:=\mathrm{g} \circ \mathrm{h}_{0}:[0,1]^{d} \hookrightarrow M$ does the job.

4.A.2. By Example 4.1 there exists a chain of prime ideals $\mathfrak{q}_{0} \subsetneq \cdots \subsetneq \mathfrak{q}_{d}:=\mathfrak{n}_{p}^{\diamond}$ in $\mathcal{S}(T)$ where $T=\operatorname{im}(\mathrm{h})$. The surjective $R$-homomorphism

$$
\varphi: \mathcal{S}^{\diamond}(M) \rightarrow \mathcal{S}(T),\left.f \mapsto f\right|_{T}
$$

provides a chain of prime ideals $\mathfrak{p}_{0} \subsetneq \cdots \subsetneq \mathfrak{p}_{d}:=\mathfrak{m}_{p}^{\diamond}$ in $\mathcal{S}^{\diamond}(M)$ where each $\mathfrak{p}_{k}:=\varphi^{-1}\left(\mathfrak{q}_{k}\right) ;$ hence, $d \leq \operatorname{ht}\left(\mathfrak{m}_{p}^{\diamond}\right) \leq \operatorname{dim}\left(\mathcal{S}^{\diamond}(M)\right) \leq d$.

4.A.3. In case $\mathcal{S}^{\diamond}(M)=\mathcal{S}(M)$, each prime ideal $\mathfrak{p}_{k}$ constructed in 4.A.2 is a $z$ ideal because it is the inverse image of the prime $z$-ideal $\mathfrak{q}_{k}$ under the surjective $R$-homomorphism $\varphi$. It follows from Theorem 1.3 and Example 4.1 that

$$
\operatorname{tr} \operatorname{deg}_{R}\left(\mathrm{qf}\left(\mathcal{S}(M) / \mathfrak{p}_{k}\right)\right)=\operatorname{tr} \operatorname{deg}_{R}\left(\mathrm{qf}\left(\mathcal{S}(T) / \mathfrak{q}_{k}\right)\right)=\mathrm{d}_{T}\left(\mathfrak{q}_{k}\right)=d-k,
$$

as required.

\section{References}

[1] Atiyah, M.F. And MacDonald, I. G.: Introduction to commutative algebra. Addison-Wesley, Reading, Mass.-London-Don Mills, Ont., 1969.

[2] Bochnak, J., Coste, M. And Roy, M.-F.: Real algebraic geometry. Ergebnisse der Mathematik und ihrer Grenzgebiete (3) 36, Springer-Verlag, Berlin, 1998.

[3] BRÖCKER, L.: Real spectra and distributions of signatures. In Real algebraic geometry and quadratic forms (Rennes, 1981), 249-272. Lecture Notes in Math. 959, Springer, Berlin-New York, 1982.

[4] Brumfiel, G. W.: The ultrafilter theorem in real algebraic geometry. Quadratic forms and real algebraic geometry (Corvallis, OR, 1986). Rocky Mountain J. Math. 19 (1989), no. 3, 611-628.

[5] Carral M. and Coste, M.: Normal spectral spaces and their dimensions. J. Pure Appl. Algebra 30 (1983), 227-235. 
[6] Cherlin, G. L. And Dickmann, M. A.: Real closed rings. I. Residue rings of rings of continuous functions. Fund. Math. 126 (1986), no. 2, 147-183.

[7] Cherlin, G. L. And Dickmann, M. A.: Real closed rings. II. Model theory. Ann. Pure Appl. Logic 25 (1983), no. 3, 213-231.

[8] Coste, M. And Roy, M.-F.: La topologie du spectre réel. In Ordered fields and real algebraic geometry (San Francisco, Calif., 1981), 27-59. Contemp. Math. 8, Amer. Math. Soc., Providence, RI, 1982.

[9] Delfs, H. And Knebusch, M.: Separation, retractions and homotopy extension in semialgebraic spaces. Pacific J. Math. 114 (1984), no. 1, 47-71.

[10] Delfs, H. And Knebusch, M.: Locally semialgebraic spaces. Lecture Notes in Mathematics 1173, Springer-Verlag, Berlin, 1985.

[11] Fernando, J. F.: On chains of prime ideals in rings of semialgebraic functions. Q. J. Math. 65 (2014), no. 3, 893-930.

[12] Fernando, J. F. And Gamboa J. M.: On Eojasiewicz's inequality and the Nullstellensatz for rings of semialgebraic functions. J. Algebra 399 (2014), 475-488.

[13] Gambon, J. M.: On prime ideals in rings of semialgebraic functions. Proc. Amer. Math. Soc. 118 (1993), no. 4, 1037-1041.

[14] Gambon, J. M. And Ruiz, J. M.: On rings of semialgebraic functions. Math. Z. 206 (1991) no. 4, 527-532.

[15] Gillman, L. and Jerison, M.: Rings of continuous functions. The University Series in Higher Mathematics, D. Van Nostrand, Princeton, NJ-Toronto-London-New York, 1960.

[16] Prestel, A. and Schwartz, N.: Model theory of real closed rings. In Valuation theory and its applications, Vol. I (Saskatoon, SK, 1999), 261-290. Fields Inst. Commun. 32, Amer. Math. Soc., Providence, RI, 2002.

[17] Schwartz, N.: Real closed rings. Habilitationsschrift, München, 1984.

[18] Schwartz, N.: Real closed rings. In Algebra and order (Luminy-Marseille, 1984), 175-194. Res. Exp. Math. 14, Heldermann, Berlin, 1986.

[19] Schwartz, N.: The basic theory of real closed spaces. Mem. Amer. Math. Soc. 77 (1989), no. 397, viii+122 pp.

[20] Schwartz, N.: Rings of continuous functions as real closed rings. In Ordered algebraic structures (Curaçao, 1995), 277-313. Kluwer Acad. Publ., Dordrecht, 1997.

[21] Schwartz, N.: Epimorphic extensions and Prüfer extensions of partially ordered rings. Manuscripta Math 102 (2000), 347-381.

[22] Schwartz, N. And Madden, J. J.: Semi-algebraic function rings and reflectors of partially ordered rings. Lecture Notes in Mathematics 1712, Springer-Verlag, Berlin, 1999.

[23] Schwartz, N. And Tressl, M.: Elementary properties of minimal and maximal points in Zariski spectra. J. Algebra 323 (2010), no. 3, 698-728.

[24] Tressl, M.: The real spectrum of continuous definable functions in o-minimal structures. Séminaire de Structures Algébriques Ordonnées 1997-1998, 68, 1999, 1-15.

[25] Tressl, M.: Super real closed rings. Fund. Math. 194 (2007), no. 2, 121-177.

[26] Tressl, M.: Bounded super real closed rings. In Logic Colloquium 2007, 220-237. Lect. Notes Log. 35, Assoc. Symbol. Logic, La Jolla, CA, 2010. 
Received June 7, 2013; revised March 4, 2014.

José F. Fernando: Departamento de Álgebra, Facultad de Ciencias Matemáticas, Universidad Complutense de Madrid, 28040 Madrid, Spain.

E-mail: josefer@mat.ucm.es

José M. Gambon: Departamento de Álgebra, Facultad de Ciencias Matemáticas, Universidad Complutense de Madrid, 28040 Madrid, Spain.

E-mail: jmgamboa@mat.ucm.es 55565 . 